\title{
Editorial
}

\section{Open Peer Reviews and Blind Peer Reviews: Merits and Demerits}

\author{
Prof. Dr. Saira Afzal
}

MBBS, MCPS, FCPS, M.Phil,PhD. Chairperson and Head, Department of Community Medicine, Editor Annals of King Edward Medical University, Lahore, Pakistan; Email: sairamust@gmail.com

DOI | http://dx.doi.org/10.21649/journal.akemu/2017/23.4.426.427

$\mathrm{T}$ he peer review process is essentially the quality control mechanism. Scientific discoveries and advancements have far reaching implications especially in health and medical publications. The quality assurance mechanism in medical journals has to be stringent and flawless. The peer review systems are continuously being criticized, debated and updated. It may be open peer review or blind peer review, both have advantages and disadvantages. Open peer review is performed for scientific quality after publication. It is also known as transparent peer review and public peer review. Open peer review is sometimes considered as the greatest pillar of scientific progression and open science. Various novel models have been developed for open peer review. Each model has its own merits and demerits. These models may include the system where the identity of both author and reviewer is known to each other. Their names, affiliations and contributions to their specialized field, already published research can be viewed for the transparency of the system. ${ }^{(1)}$ For still others, the review reports of the reviewers are also published alongside the research articles. In some situations, only invited experts are allowed to give their review reports. These experts in specialized fields are usually decided on merit by the editorial team. Sometimes, it's the combination of different models for open peer review. Various scientific journals experimented with open peer review to improve the quality of publication. One such example is "Nature", where an option is provided to the authors of having their research articles published online during the peer review process with the reviewer reports. The names and affiliations of authors and re- viewers were also disclosed. Only 5\% of the authors opted for an open peer review and only few online research received comments from the experts in similar specialty. Other demerits include failure to get precise and focused comments on the research article, low quality reviews, dissatisfaction among the authors after peer review.

Thus it was proposed on various scientific forums that definition of open peer review should be revisited and it should adapt different models effectively, including publishing review reports alongside the research article, making both reviewers and authors identities open and inviting the comments of the experts in specific field. The open science cannot progress without encouraging the greater participation of authors and reviewers in the peer review process. Incentives and rewards should be planned for increasing the trend towards open peer review process.

In double blind peer review system both author and reviewer are anonymous to each other. In single blind the identity of the reviewer is hidden from the author. In medical sciences there is greater concern about human health and any discovery or invention has long lasting effect on humans, thus an infallible system of blind peer review is required. The blind peer review decreased the bias caused by the reviewer's personnel and scientific beliefs. Sometimes the reviewer couldn't separate them from geographical, racial or institutional prejudices. The conflict of interests may cause rejection of a very innovative research, if the blind peer review is not followed. Thus double blind 
peer review gives more credit to the methodology and evidence produced than the researcher's profile. However, there is an argument that in double blind peer review the reviewers can guess the researcher's profile by looking at the citations or references. Sometimes little effort and hard work from the reviewers resulted in serious errors in methodology of published medical research. These errors can lead to harmful effects on the well being of the individuals. The poor selection of blind peer reviewers by the editorial team, time investment and management problems by the reviewers and authors, transparency issues, and failure to implement the uniform standards during blind peer review process are some important points that has produced criticism on this system. ${ }^{(2)}$

Nevertheless, peer review is continuously favored despite all the disadvantages as it has produced acceptable discoveries, evidence based practices and good quality publications for scientific advancements.
The role of the author is pivotal who is required to produce the manuscript according to the comments of the peer reviewers. They can improve their work by reflection and positive criticism from the peers. The reviewers should avoid subjective comments and maintain the highest possible standards of transparent decisions within specified time. The peer review systems either open or blind has proven to enhance the quality of publications for scientific progress.

\section{References}

1. Watson R. Peer review under the spotlight in the UK. Journal of Advanced Nursing. 2012; 68(4): 718-720.

2. Jefferson T, Alderson P, Wager E, Davidoff F. Effects of editorial peer review: a systematic review. Journal of the American Medical Association.2002; 287,:2784-6. 